\title{
Fourier-domain digital holographic optical coherence imaging of living tissue
}

\author{
Kwan Jeong, ${ }^{1, \star}$ John J. Turek, ${ }^{2}$ and David D. Nolte ${ }^{1}$ \\ ${ }^{1}$ Department of Physics, Purdue University, West Lafayette, Indiana 47907-2036, USA \\ 2Department of Basic Medical Sciences, Purdue University, West Lafayette, Indiana 47907, USA \\ ${ }^{*}$ Corresponding author: jeongk@physics.purdue.edu \\ Received 18 December 2006; revised 11 April 2007; accepted 20 April 2007; \\ posted 25 April 2007 (Doc. ID 78068); published 6 July 2007
}

\begin{abstract}
Digital holographic optical coherence imaging is a full-frame coherence-gated imaging approach that uses a CCD camera to record and reconstruct digital holograms from living tissue. Recording digital holograms at the optical Fourier plane has advantages for diffuse targets compared with Fresnel off-axis digital holography. A digital hologram captured at the Fourier plane requires only a 2D fast Fourier transform for numerical reconstruction. We have applied this technique for the depth-resolved imaging of rat osteogenic tumor multicellular spheroids and acquired cross-section images of the anterior segment and the retinal region of a mouse eye. A penetration depth of $1.4 \mathrm{~mm}$ for the tumor spheroids was achieved. (C) 2007 Optical Society of America

OCIS codes: $170.1650,090.1760,070.2590,110.6150,170.7050$.
\end{abstract}

\section{Introduction}

Holography uses a coherent light source to record and reconstruct the amplitude and phase information of a wavefront through interference between an object wave and a reference wave. One application of holography is for coherence-gated imaging through turbid media using short-coherence light. Stetson first showed the principle of coherence gating for imaging through a foglike medium [1]. In 1989, light-inflight holography was demonstrated, exploiting the finite coherence length of the light source to facilitate the depth-resolved imaging of 3D objects [2]. However, photographic film was used as the holographic recording media. Subsequent research has concentrated on the development of real-time methods to perform hologram recording and its reconstruction, focusing mainly on two techniques: photorefractive holography and digital holography.

Photorefractive holography uses a photorefractive material as a recording medium for real-time imaging. It was demonstrated initially in a photorefractive crystal $[3,4]$, and photorefractive quantum well (PRQW) devices were found to be best suited for this approach

0003-6935/07/224999-10\$15.00/0

(C) 2007 Optical Society of America
[5]. Photorefractive holography using short-coherence light has been used in depth-resolved imaging inside living tissue, such as rat osteogenic tumor spheroids and in the mouse eye [6,7]. Photorefractive holography is attractive for real-time imaging because it is a noncomputational background-free direct-imaging technique, and it has adaptive advantages using the fast response time of dynamic holographic films, which permits the use of a vibrating diffuser to reduce multiply scattered light [8]. However, sensitivity in photorefractive holography is limited by the diffraction efficiency (with a typical value of $10^{-3}$ ) of the PRQW device [9], and the longitudinal resolution is limited by its spectral range (typically $5 \mathrm{~nm}$ ).

In digital holography, the dynamic holographic film is replaced by a charge-coupled device (CCD) array, and the holographic reconstruction is performed numerically operating on the digital hologram recorded on the CCD array. Because a CCD sensor has a high bit range and wide spectral bandwidth, an improvement in sensitivity and resolution can be accomplished at the cost of only requiring computational reconstruction. Numerical hologram reconstruction was initiated in the early 1970s [10,11], and digital holography was developed as a fast real-time imaging technique in the 1990s $[12,13]$ in various applications such as phase- 
shifting digital holography [14], phase-contrast imaging $[15,16]$, shape measurement [17], encrypting information [18], and refractive index tomography [19], thanks to the improvement of CCD sensitivity and advances in computer performance. Digital holography using short-coherence light [20-22] was developed for three-dimensional microscopy, and time-domain optical coherence tomography with digital holographic microscopy was demonstrated with an application to ophthalmology [23]. Depth-gated imaging in digital holography was also achieved using multiwavelength scanning digital holography $[24,25]$.

In the previous demonstrations of digital holography, the CCD camera was located between the Fourier plane and the image plane in a Fresnel off-axis regime, which is known as Fresnel off-axis digital holography. This requires the Fresnel approximation in numerical reconstruction algorithms to reconstruct the focused image. However, the comparative ease of hologram recording and numerical reconstruction can be gained by placing the CCD camera at the Fourier plane, which is called Fourier-domain digital holography (FDDH). In photorefractive holography, it was demonstrated that Fourier-domain holography for diffuse targets has a higher sensitivity compared with image-domain holography in which the holographic film is placed at the image plane $[7,26]$. In digital holography, FDDH was demonstrated recently in a transmission geometry using a cw He-Ne laser for a biological phantom [27]. When short-coherence light is used in FDDH, coherencegated depth-resolved imaging can be achieved, which we call Fourier-domain digital holographic optical coherence imaging (FD-DHOCI).

The chief advantages of FDDH are a simple numerical reconstruction algorithm, digital Fourier filtering capacity, and high sensitivity. Because the digital hologram is recorded at the Fourier plane, a digital Fourier transform of the digital hologram reconstructs the focused image. Therefore the reconstruction algorithm in FDDH is simply a fast Fourier transform (FFT), which enables faster numerical reconstruction than for the algorithm using the Fresnel approximation. Fourier filtering such as high-pass filtering, dark-field imaging, and phase-contrast imaging can be applied easily to the digital hologram without extra lenses. Sensitivity in digital holography is mainly determined by the CCD bit range and the CCD sensitivity. The improvement of CCD technology offers higher sensitivity in FDDH. Furthermore, FDDH has a multiplex advantage in sensitivity due to parallel detection compared with a pointscanning serial detection such as time-domain optical coherence tomography [28].

Because of these advantages, it is attractive to investigate FD-DHOCI systems using short-coherence light as a candidate for biological depth-gated imaging. In this paper we present a detailed study of the FD-DHOCI system. This is a wide-field coherencegated imaging approach using FDDH, which acquires en face images at successive depths inside scattering media. In Section 2, we present a detailed description of the FD-DHOCI system, including the basic theory, the experimental setup, and the principle of numerical reconstruction. Section 3 describes resolution and sensitivity in the FD-DHOCI system. In Section 4 , we show experimental results for tumor spheroids and mouse eyes.

\section{Fourier-Domain Digital Holography Principle}

\section{A. General Principles}

The basic configuration of digital recording in FDDH is illustrated in Fig. 1. Consider a coherent source that produces a reference plane wave and an object wave. The object wave $\psi_{O}(x, y)$ at the object plane is optically Fourier-transformed by a lens with focal length $f$. For an ideal lens, the object wave at the Fourier plane is given by

$$
\begin{aligned}
\psi_{O F}\left(x^{\prime}, y^{\prime}\right)= & \frac{i}{\lambda f} \exp \left(-\frac{i 4 \pi f}{\lambda}\right) F\left(\frac{x^{\prime}}{\lambda f}, \frac{y^{\prime}}{\lambda f}\right) \\
= & \frac{i}{\lambda f} \exp \left(-\frac{i 4 \pi f}{\lambda}\right) \int_{-\infty}^{\infty} \int_{-\infty}^{\infty} \psi_{O}(x, y) \\
& \times \exp \left(\frac{i 2 \pi}{\lambda f}\left(x x^{\prime}+y y^{\prime}\right)\right) \mathrm{d} x \mathrm{~d} y,
\end{aligned}
$$

where $\lambda$ is the wavelength of the source and $F$ is the Fourier transform. Consider the reference plane wave given by

$$
\psi_{R}=\psi_{R 0} \exp \left\lfloor i 2 \pi\left(v_{x 0} x+v_{y 0} y\right)\right\rfloor,
$$

where $v_{x 0}$ and $v_{y 0}$ are the spatial wavenumbers. When the object and the reference waves are zero-pathmatched at the Fourier plane by adjusting the path length of the reference wave, the intensity of the hologram is given by

$I_{H}\left(x^{\prime}, y^{\prime}\right)=\left|\psi_{R}\right|^{2}+\left|\psi_{O F}\right|^{2}+\psi_{R}^{*} \psi_{O F}+\psi_{R} \psi_{O F} *$.

The intensity $I_{H}\left(x^{\prime}, y^{\prime}\right)$ is physically recorded with a photographic or photorefractive film in conventional

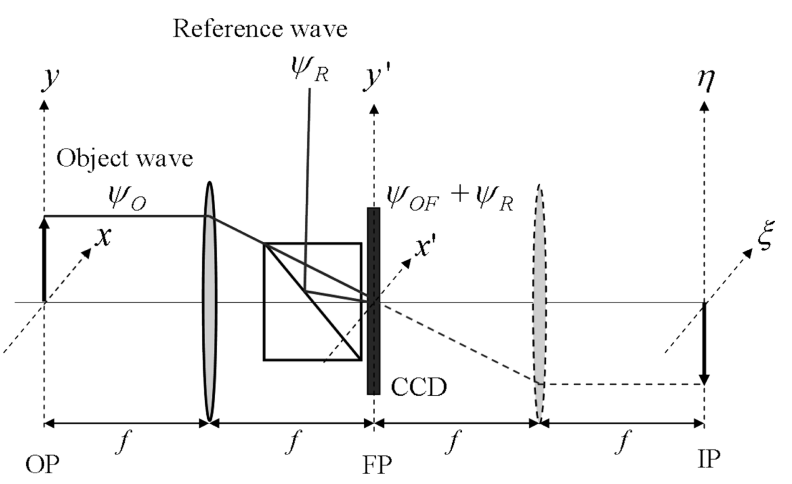

Fig. 1. Optical setup for FDDH: OP, object plane; FP, Fourier plane; IP, image plane; $f$, focal length of the lens; $\psi_{O}$, object wave at OP; $\psi_{O F}$, object wave at FP; $\psi_{R}$, reference wave. The role of the reconstruction lens is replaced by numerical reconstruction of the CCD data. 
holography, but this is recorded electronically by a CCD camera in digital holography. The digital hologram is numerically reconstructed using numerical reconstruction algorithms, while in conventional holography the physical hologram is reconstructed by illuminating the film with the same reference wave $\psi_{R}$. In FDDH, a simple Fourier transform is used to reconstruct the digital hologram because the digital hologram is recorded at the Fourier plane. The numerical Fourier transform of the digital hologram is analogous with an optical Fourier transform performed by a second lens. The Fourier transform of the intensity $I_{H}\left(x^{\prime}, y^{\prime}\right)$ is given by

$$
\begin{aligned}
F T\left(I_{H}\right)= & F T\left(\left|\psi_{R}\right|^{2}\right)+F T\left(\left|\psi_{O F}\right|^{2}\right)+F T\left(\psi_{R}^{*} \psi_{O F}\right) \\
& +F T\left(\psi_{R} \psi_{O F}{ }^{*}\right) \equiv F_{1}+F_{2}+F_{3}+F_{4} .
\end{aligned}
$$

In Eq. (4), the third term produces a holographic image, and the fourth term produces a conjugate holographic image, while the first term and second term produce a zero-order image. The third term and the fourth term in Eq. (4) can be calculated using Eqs. (1) and (2), which are given by

$$
\begin{aligned}
F_{3}\left(v_{x^{\prime}}, v_{y^{\prime}}\right)= & i \lambda f \psi_{R 0} \exp \left(-\frac{i 4 \pi f}{\lambda}\right) \psi_{O}\left(-\lambda f v_{x^{\prime}}+\lambda f v_{x 0},\right. \\
& \left.-\lambda f v_{y^{\prime}}+\lambda f v_{y 0}\right), \\
F_{4}\left(v_{x^{\prime}}, v_{y^{\prime}}\right)= & -i \lambda f \psi_{R 0} \exp \left(\frac{i 4 \pi f}{\lambda}\right) \psi_{O} *\left(\lambda f v_{x^{\prime}}+\lambda f v_{x 0},\right. \\
& \left.\lambda f v_{y^{\prime}}+\lambda f v_{y 0}\right) .
\end{aligned}
$$

The holographic image $F_{3}$ is located at $\left(\lambda f v_{x 0}, \lambda f v_{y 0}\right)$ and the conjugate image $F_{4}$ is located at $\left(-\lambda f v_{x 0}\right.$, $-\lambda f v_{y 0}$ ), which are spatially separated from the zeroorder image located at $(0,0)$. Therefore, the numerical Fourier transformation of the digital hologram acquired at the Fourier plane provides both amplitude and phase information about the object.

\section{B. Experimental Setup}

We used the experimental setup shown in Fig. 2 to record and reconstruct digital holograms using a mode-locked Ti:sapphire laser (100 fs pulse duration, $100 \mathrm{MHz}$ repetition rate). The center wavelength of the laser was $840 \mathrm{~nm}$ with a bandwidth of $17 \mathrm{~nm}$. The output beam from the laser passed through an initial polarizing beam splitter (PBS) to produce an object beam with a vertical polarization and a reference beam with a horizontal polarization. Using a neutral density filter and a half-wave plate before the first PBS, we controlled the incoming beam intensity and the beam ratio of the object beam to the reference beam. A quarter-wave plate behind the second PBS ensured that the backscattered object beam had horizontal polarization after returning through the quarter-wave plate. After the backscattered object beam passed the second PBS, the object beam was relayed with a 1:1 magnification by lenses $\mathrm{L} 1$ and L2 (with focal lengths of $15 \mathrm{~cm})$. The lens L3 $(4.8 \mathrm{~cm}$

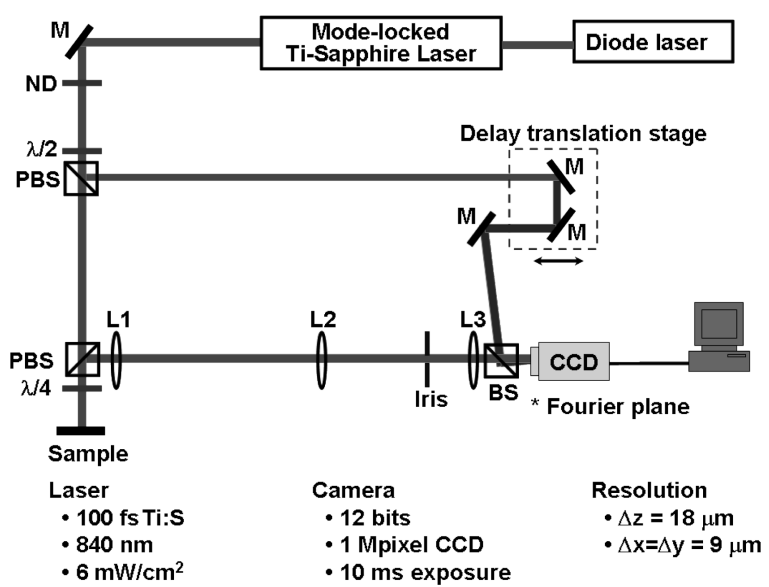

Fig. 2. Experimental setup for the FDDH. M's, mirrors; ND, neutral density filter; PBSs, polarizing beam splitters; BS, beam splitter; L1-L3, lenses; $\lambda / 2$, half-wave plate; $\lambda / 4$, quarter-wave plate.

focal length) performed the Fourier transform of the object beam located at the CCD camera, where it interfered with the zero-path-matched reference beam that passed through the computer-controlled delay line. The CCD camera is placed at the Fourier plane of the object by adjusting a translation stage at the object plane. The fringe spacing was adjusted to be three times the size of a pixel. The interference fringes between the object beam and the reference beam were recorded by the CCD camera and the computer. Digital holograms were reconstructed by the FFT. The typical object intensity for living tissue at the object plane was $6 \mathrm{~mW} / \mathrm{mm}^{2}$ and an 8-bit or a 12-bit CCD camera with 1 megapixel resolution was used with an exposure time of $10 \mathrm{~ms}$.

\section{Numerical Reconstruction and Zero-Order Suppression}

Consider a digital hologram recorded by the CCD chip with a pixel size of $\Delta x^{\prime}\left(\Delta x^{\prime}=\Delta y^{\prime}\right)$ and $N \times N$ pixels. The area of the CCD is given by $L \times L$ $\left(L=N \Delta x^{\prime}\right)$, where $\Delta x^{\prime}$ and $\Delta y^{\prime}$ are the sampling intervals at the hologram plane. The numerical reconstruction algorithm in FDDH is the FFT. After the FFT of the digital hologram, the reconstructed image has $N \times N$ pixels with a pixel size $\Delta \xi(\Delta \xi=\Delta \eta)$ given by

$$
\Delta \xi=\Delta \eta=\lambda f \Delta v_{x^{\prime}}=\lambda f \Delta v_{y^{\prime}}=\frac{\lambda f}{L}
$$

where $\Delta v_{x^{\prime}}\left(\Delta v_{x^{\prime}}=\Delta v_{y^{\prime}}=1 / L\right)$ is the sampling interval spatial frequency.

A typical digital hologram is shown in Fig. 3, which was recorded on the CCD by a short-coherence source (Ti:sapphire) using a rat tumor spheroid as a target. The digital hologram containing $N \times N=800 \times$ 800 pixels was encoded with 4096 gray levels (12 bits). The pixel size is $\Delta x^{\prime}=\Delta y^{\prime}=6.8 \mu \mathrm{m}$ and the area of the CCD chip is $L \times L=5.44 \mathrm{~mm} \times$ $5.44 \mathrm{~mm}$. The enlargement of the rectangular section 

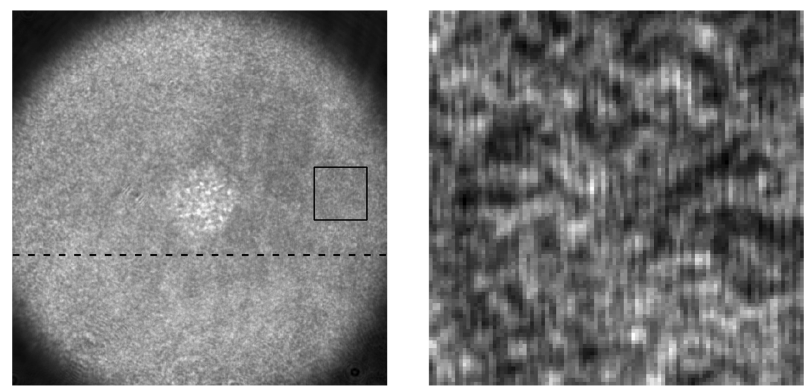

Fig. 3. Typical digital hologram (left) and the enlargement of the rectangular section (right). Interference between the object wave and the reference wave is shown. The fringe spacing is three times the size of a pixel. The interference pattern (visibility) is weak because highly scattering living tissue was used as a target.

in Fig. 3 shows interference fringes between the reference wave and the object wave. Note that the contrast of the interference pattern (visibility) is weak because of the high scattering by the living tissue target, making the background much brighter than the interference fringes.

The dashed line in Fig. 3 was selected to illustrate the process of hologram reconstruction in one dimension, and its intensity as a function of position is shown in Fig. 4(a). The hologram reconstruction of Fig. 4(a) is shown in Fig. 4(b), which is the spectral power as a function of spatial frequency. In Fig. 4(b), the holographic image and its conjugate image are the sidebands, and the zero-order image is at the center. The position of the sidebands is determined by

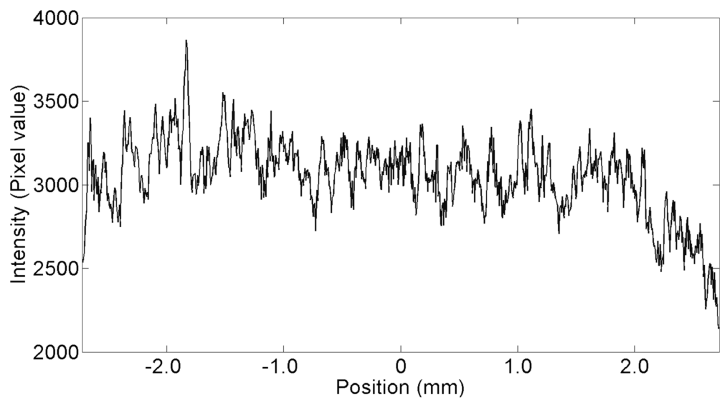

(a)

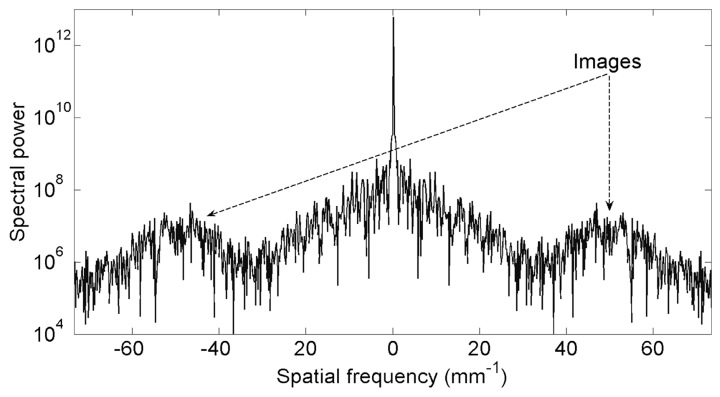

(b)

Fig. 4. Digital hologram and its reconstruction in one dimension. (a) Intensity as a function of position for the dashed line in Fig. 3 and (b) its reconstruction by the FFT, which shows the zero-order image (center) and holographic images (sidebands). the fringe spacing. To record interference fringes in the digital hologram, the fringe spacing should range from twice the pixel size (minimum) to the CCD chip size (in-line holography). The spatial frequency corresponding to the maximum fringe spacing is $1 / L$ $=\Delta v_{x^{\prime}}$, and the spatial frequency for the minimum fringe spacing is $1 /\left(2 \Delta x^{\prime}\right)=N \Delta v_{x^{\prime}} / 2$, which is the spatial frequency limit. Four times the pixel size $\left(4 \Delta x^{\prime}\right)$ is the best fringe spacing when the sideband is located at half of the spatial frequency limit. When the fringe spacing is $4 \Delta x^{\prime}$, the maximum field of view for the holographic image is achieved with a size $N \Delta \xi / 2=\lambda f /\left(2 \Delta x^{\prime}\right)$. The fringe spacing for Fig. 3 was $3 \Delta x^{\prime}$ with the center of the sideband located at the spatial frequency of $1 /\left(3 \Delta x^{\prime}\right)=49 \mathrm{~mm}^{-1}$ in Fig. 4(b).

An example of the numerical reconstruction in 2D is shown in Fig. 5(a). The target was diffuse white paper with a reflective letter " $R$ " in a circle. For this holographic image, we recorded a digital hologram using the short-coherence source with a zero-pathmatched reference, and then performed the FFT of the digital hologram. The holographic image and its conjugate image are spatially separated from the zero-order image in the center area. However, the zero-order image overlaps the holographic image, and this increases the background in the reconstructed image. A simple method for zero-order suppression is to capture the non-zero-path-matched intensity without interference fringes. In FDDHOCI, the non-zero-path-matched intensity is ob-

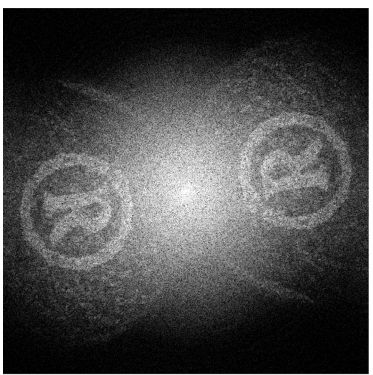

(a)

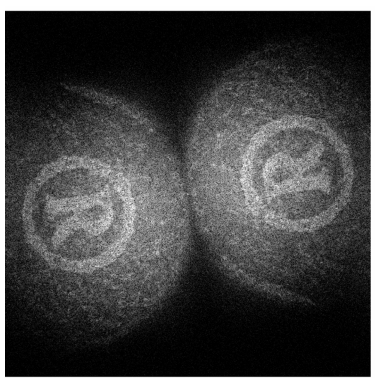

(c)

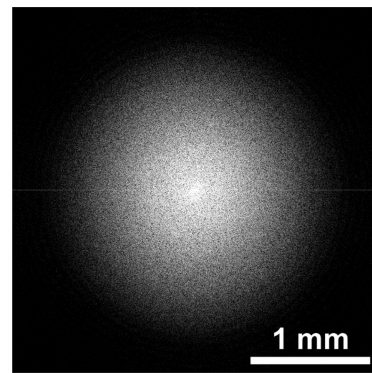

(b)

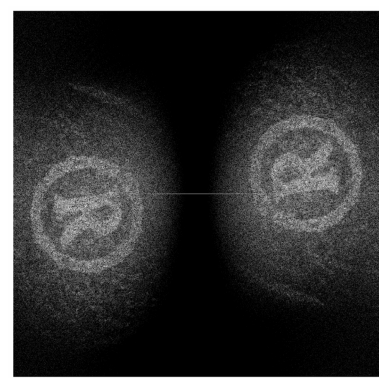

(d)
Fig. 5. Reconstructions of (a) a zero-path-matched digital hologram and (b) a non-zero-path-matched digital hologram of diffuse paper with the letter " $R$ " in a circle on a log reflectance scale. Zero-order suppressed holographic images are shown in (c) for the non-zero-path-matched intensity subtraction method, and in (d) for the 3-pixel-averaged intensity subtraction method. 
tained by changing the path of the reference wave. The reconstructed image for the digital hologram without interference fringes is shown in Fig. 5(b), in which only the zero-order image is shown. The reconstructed image with zero-order suppression is shown in Fig. 5(c), where the FFT was performed after subtraction of the non-zero-path-matched digital hologram from the zero-path-matched digital hologram. This zero-order suppression method by subtracting the non-zero-path-matched intensity requires higher experimental effort due to the additional measurement, and is less effective for a moving target because the zero-order image may change due to the movement of the target during the measurement.

We also took a second approach to suppress the zeroorder image by averaging the interference fringes of a single acquisition. A zero-order image is obtained when the digital hologram has no interference. We approximated a digital hologram without interference by averaging the interference fringes over the fringe spacing interval. The fringe spacing for Fig. 3 was $3 \Delta x^{\prime}$ and therefore the digital hologram without interference can be approximated by 3-pixel averaging in the horizontal direction. An example using the line plot in Fig. 4(a) is shown in Fig. 6. Figure 6(a) shows the intensity plot where the 3-pixel-averaged intensity was subtracted from the curve plot in Fig. 6(a). The spatial spectrum of Fig. 6(a) is shown by a solid curve

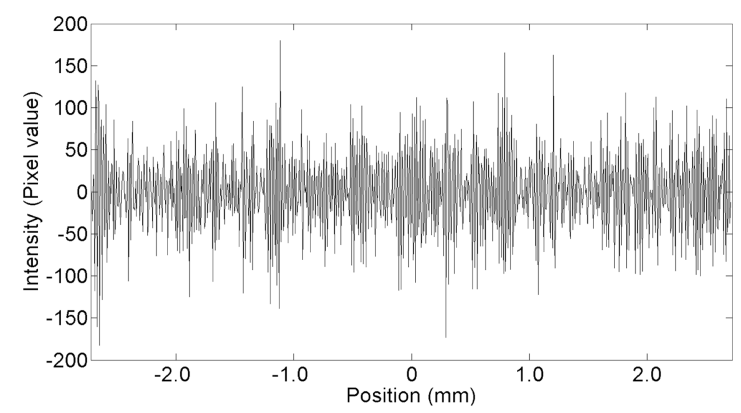

(a)

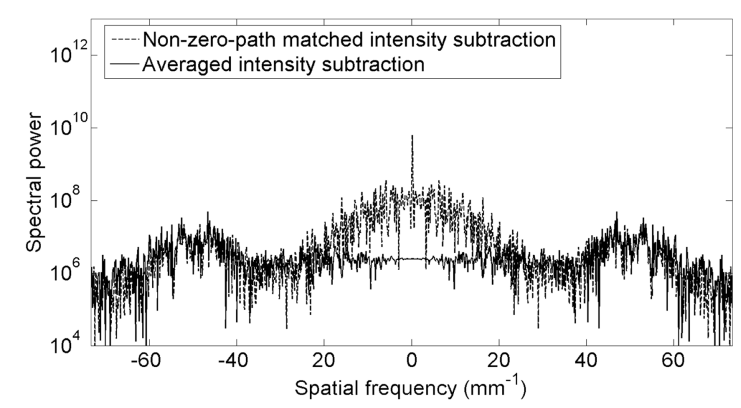

(b)

Fig. 6. (a) Intensity as a function of position after the 3-pixelaveraged intensity was subtracted from the intensity plot in Fig. 3(a). (b) Zero-order suppressed spatial spectrum as a function of spatial frequency. The solid curve is for the zero-order suppression by the 3-pixel-averaged intensity subtraction method, and the dashed curve is for the zero-order suppression by the non-zeropath-matched intensity subtraction. in Fig. 6(b). The spatial spectrum for the zero-order suppression by the non-zero-path-matched intensity subtraction method is shown by a dashed curve in Fig. 6(b) to compare the two zero-order suppression methods. A considerable amount of zero-order background remains after zero-order suppression by the non-zeropath-matched intensity subtraction method, but most of the zero-order image was removed after zero-order suppression by the 3-pixel-averaged intensity subtraction.

An example in two dimensions is shown in Fig. $5(\mathrm{~d})$, which is the zero-order suppressed image using the 3-pixel-averaged intensity subtraction method. This method has the side effect of suppressing the desired holographic image near the zero-order image in addition to the unwanted zero-order image, which changes the spatial distribution of the holographic image intensity. Therefore, the non-zero-pathmatched intensity subtraction method maintained better image fidelity than the 3-pixel-averaged intensity subtraction method, while the 3-pixel-averaged intensity subtraction method, with no need for additional measurement, can be used effectively when the zero-order image is changing with time.

\section{Resolution and Sensitivity}

The transverse resolution in FD-DHOCI depends on the area of the CCD chip. If the object beam at the Fourier plane covers the entire area of the CCD, then the transverse resolution at the Rayleigh criterion is

$$
R_{s}=1.22 \lambda f / L=1.22 \Delta \xi
$$

The longitudinal resolution depends on the coherence length of the short-coherence source. The longitudinal resolution is given by [29]:

$$
\Delta z=\ln (2) \frac{2}{\pi} \frac{\lambda^{2}}{\Delta \lambda}
$$

where $\Delta \lambda$ is the FWHM wavelength bandwidth of the source. The measured $\Delta \lambda$ was $17 \mathrm{~nm}$. We used a 12-bit CCD camera with $\Delta x^{\prime}=6.8 \mu \mathrm{m}, N=800$, $\lambda=840 \mathrm{~nm}$, and $f=4.8 \mathrm{~cm}$ for the data in this paper. The transverse and the longitudinal resolutions for this system are calculated to be 9.0 and $18.3 \mu \mathrm{m}$, respectively.

To measure the transverse resolution, we used a specular U.S. Air Force (USAF) test chart. For the specular test chart, the intensity at the Fourier plane was localized near the center of the spectrum, which saturates the CCD camera, while the intensity for diffuse targets is uniformly distributed at the Fourier plane. To make a relatively uniform distribution of intensity at the Fourier plane, we sequentially shifted a vibrating $5^{\circ}$ diffuser and recorded 100 digital holograms. These digital holograms were numerically reconstructed using the 3-pixel-averaging zero-order suppression method, and then averaged over 100 reconstructed images, shown in Fig. 7. The sixth element 

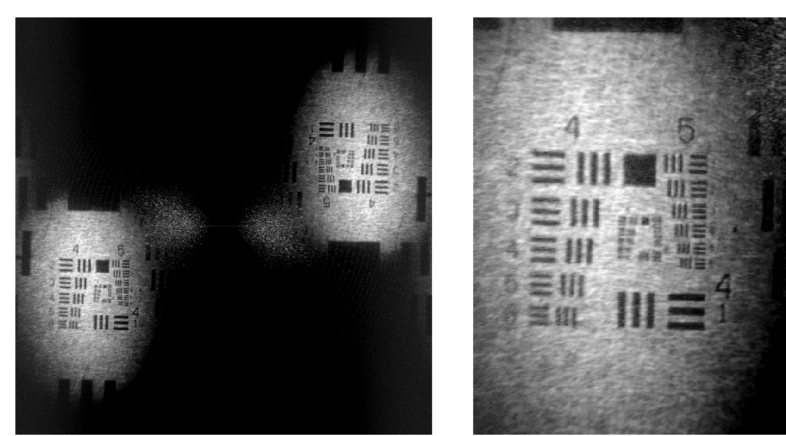

Fig. 7. Reconstructed image of a USAF test chart (left) and its enlargement (right), which is the average of 100 sequential digital holographic images using the 3-pixel-averaging zero-order suppression method. A vibrating $5^{\circ}$ diffuser was used to make a uniform intensity distribution at the Fourier plane.

of group 5 of the USAF test target can be resolved in Fig. 7 with a bar size of $8.8 \mu \mathrm{m}$.

In FD-DHOCI, the CCD camera should be placed at the Fourier plane in the range of the depth of focus of the system. The formula for the depth of focus is given by [30]:

$$
\Delta z=\frac{\lambda}{2 N A^{2}}
$$

where $\lambda$ is the wavelength, and $N A$ is the numerical aperture. The depth of focus for a system with a transverse resolution of $9.0 \mu \mathrm{m}$ is calculated to be $131 \mu \mathrm{m}$. We used the translation stage at the object plane to center the target within the depth of focus. After placing the target at the focal plane, digital holograms were captured by moving the translation stage at the object plane when the size of the target is larger than the depth of focus.

When imaging a 2D target with short coherence light, the holographic volume containing fringes is limited depending on the coherence length and the angle between the object and reference waves, which is known as the "walk-off" effect [31]. The walk-off effect can be avoided by tilting the target, with the cost of decreased resolution by increasing the distance of the parts of the object plane from the focal plane, as illustrated in Fig. 8. When the coherence length is $l_{C}$ and the angle between the object and reference waves is $\theta$, the fringe width $W_{\mathrm{Fv}}$ is $l_{C} / \sin \theta$. The 2D target tilted with an angle of $\theta$ results in a maximum out-of-focus of $0.5 W_{T} \tan \theta$ at the edge of the target image, where $W_{T}$ is the size of the target. In contrast to $2 \mathrm{D}$, holograms for a $3 \mathrm{D}$ target intrinsically suppress the fringe walk-off, resulting in a maximum out-of-focus of $0.5 W_{T} \tan \theta$ for all target images. In our FD-DHOCI system, the angle $\theta$ is $2.36^{\circ}$ and the typical size of the reconstructed image is $1 \mathrm{~mm}$, which gives the maximum out-of-focus of $21 \mu \mathrm{m}$. The out-of-focus is approximately a factor of 6 smaller than the depth of focus in our FD-DHOCI system. Therefore, the transverse resolution is determined by the NA in the current system. However, the out-of-

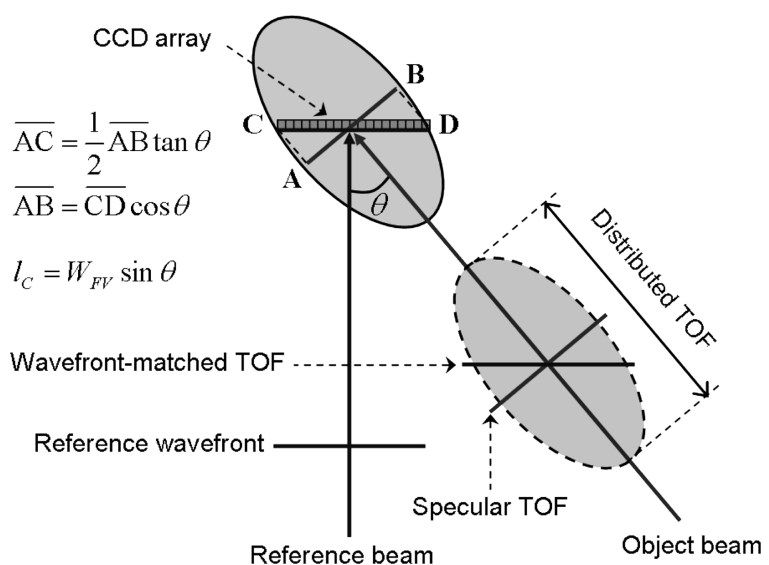

Fig. 8. Illustration of fringe walk-off in a $2 \mathrm{D}$ target and the outof-focus in a $3 \mathrm{D}$ target. The fringe width $W_{\mathrm{FV}}$ depends on the coherence length $l_{C}$ and the angle $\theta$ between the reference and object waves. The fringe walk-off is suppressed for a $3 \mathrm{D}$ target with the cost of increased out-of-focus. TOF, time-of-flight.

focus may present challenges for the development of a ultrahigh resolution FD-DHOCI system. For example, the system with $2 \mu \mathrm{m}$ transverse resolution has a depth of focus of $6.4 \mu \mathrm{m}$, which allows a reconstructed image size of $311 \mu \mathrm{m}$, but requires a decrease in the angle between the reference and object waves to increase the field of view.

To measure longitudinal resolution and sensitivity in FD-DHOCI, we used a 3.0 neutral density filter followed by a mirror as a target, which gave a reflectivity of $-60 \mathrm{~dB}$. Digital holograms for the mirror were captured by the CCD camera through the computer-controlled delay stage with a depth step of $2 \mu \mathrm{m}$. Figure 9(a) shows the average of 100 pseudoA-scans selected from holographic en face images after the digital reconstruction. The incident object intensity was $5 \mathrm{~mW} / \mathrm{mm}^{2}$, and the exposure time of the CCD camera was 10 ms. The FWHM in Fig. 9(a) is $18.2 \mu \mathrm{m}$, which agrees with the calculated value from Eq. (9). The transverse resolution can be improved by using a shorter focal length lens and a CCD with a larger chip size. The longitudinal resolution can be improved by using a shorter coherence length.

When the sample has reflectivity of $R_{s}$ and the maximum digital number for the sample after the FFT of the digital hologram is $V_{s, \max }$, the sensitivity $S$ in the decibel of the system is given by

$$
S=20 \log \frac{V_{s, \max }}{\sigma_{n}}+10 \log \frac{1}{R_{s}},
$$

where $\sigma_{n}$ is the standard deviation of the digital number on all voxels (without a target) after the FFT. We used a target with reflectivity of $-60 \mathrm{~dB}$ to measure the system sensitivity. The value of $V_{s, \max } / \sigma_{n}$ was 20.75 , and the sensitivity was measured to be $86 \mathrm{~dB}$. This demonstrates that the FD-DHOCI system has a sensitivity of $86 \mathrm{~dB}$ for an object intensity of $5 \mathrm{~mW} / \mathrm{mm}^{2}$ and an exposure time of $10 \mathrm{~ms}$ with the experimental setup in Fig. 2. Figure 9(b) shows the 


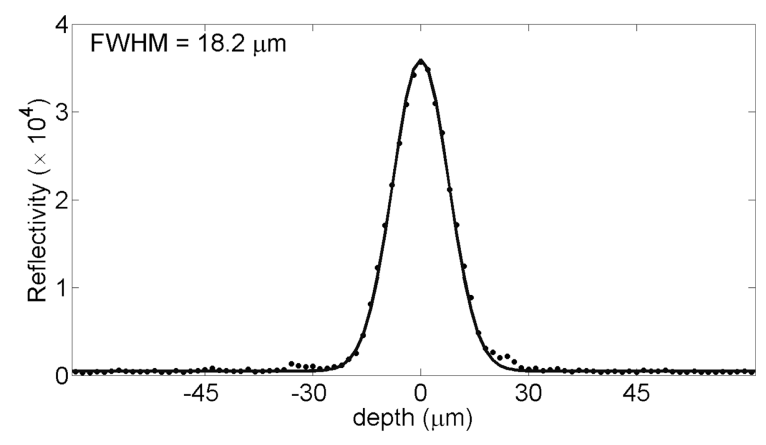

(a)

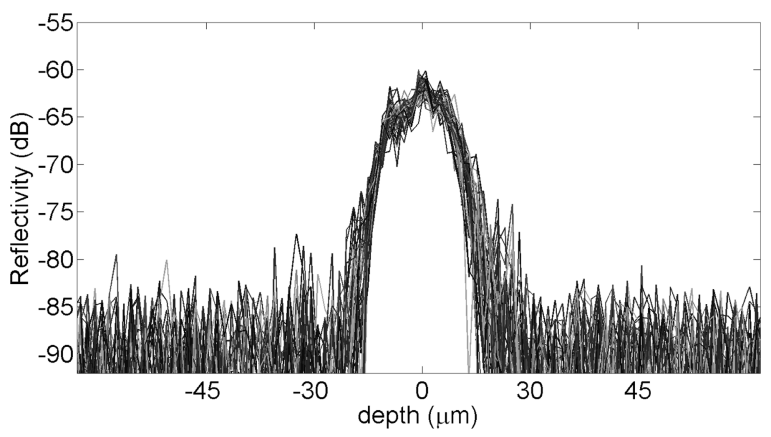

(b)

Fig. 9. (a) Linear plot of reflectivity as a function of depth (averaged over $100 \mathrm{~A}$-scans selected from holographic en face images of a planar target with a reflectivity of $-60 \mathrm{~dB}$ ), where the FWHM is $18.2 \mu \mathrm{m}$. (b) Logarithmic plot of 20 pseudo-A-scans, which shows the measured sensitivity near $-86 \mathrm{~dB}$.

logarithmic plot of 20 pseudo-A-scans selected from holographic en face images of the planar target. The noise floor agrees with the measured system sensitivity.

\section{Experimental Results for Rat Tumor Spheroids and Mouse Eye}

To study the performance of FD-DHOCI in tissue, we used rat osteogenic sarcoma tumor spheroids that were grown in vitro. Rat osteogenic sarcoma UMR106 cells are cultured in a rotating bioreactor where they are maintained in suspension. The spheroids may be grown up to several millimeters in diameter, and are thus large enough to simulate the thickness of different mammalian tissue (skin epidermis is $70-120 \mu \mathrm{m}$ in thickness over most of the human body). An advantage to using this continuous culture model is that fresh spheroids of varying size are easily prepared on a daily basis. Overall, the tumor spheroids provide a reasonable tissue model that does not require special handling of animal subjects [32]. As tumor spheroids are cultured, they undergo cell apoptosis or necrosis in their center and so consist of an inner necrotic core and an outer shell with a 100 to $200 \mu \mathrm{m}$ thickness of healthy cells [33].

To obtain stacks of images of the internal structure of tumor spheroids, we captured digital holograms through the computer-controlled reference delay with a depth step of $10 \mu \mathrm{m}$. We used the reference delay stage instead of the signal delay stage to avoid the vibration of the tumor growth medium in a Petri dish. The depth of focus was approximately $1 \mathrm{~mm}$ at a depth of $400 \mu \mathrm{m}$ for a tumor spheroid with $800 \mu \mathrm{m}$ diameter, which makes the out-of-focus effect by the use of the reference delay stage negligible. The captured digital holograms were Fourier-transformed and zero-order-subtracted. This data acquisition method is called a fly-through. Figure 10(a) shows $x-y$ sections that were selected per every eight depths from en face holographic images for a healthy tumor

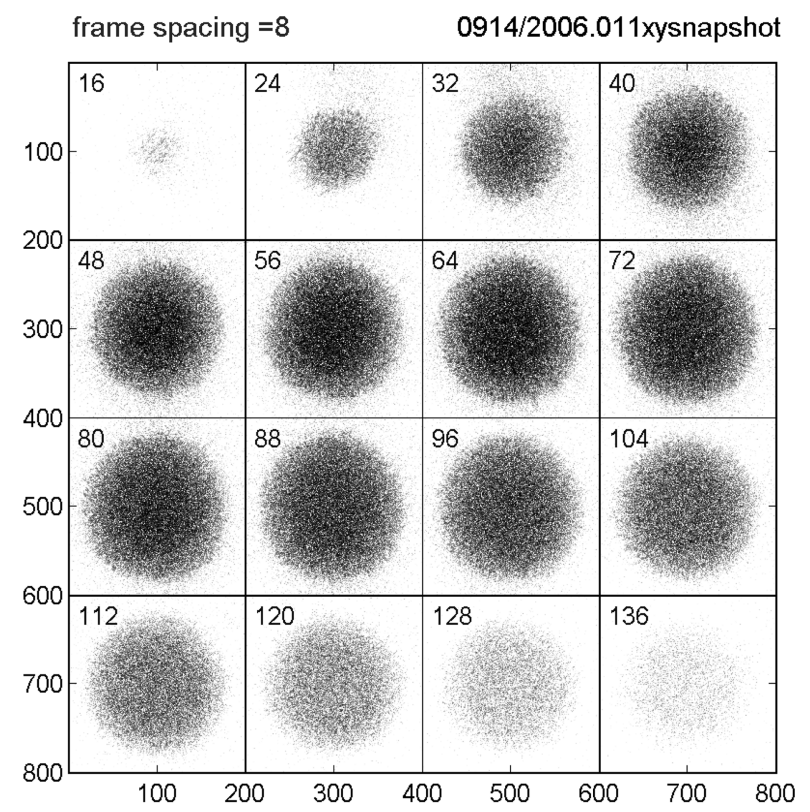

(a)

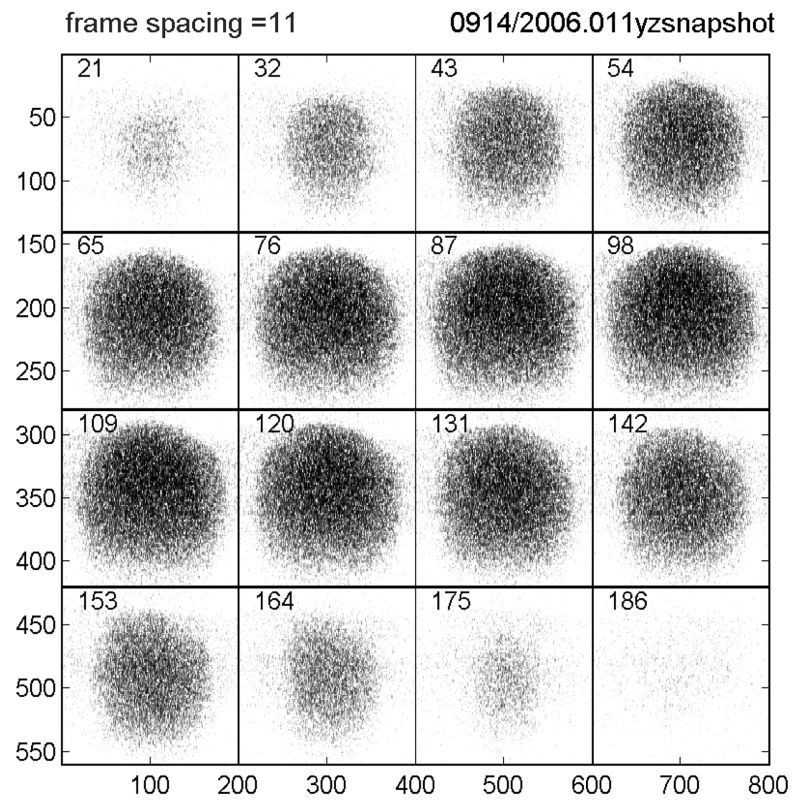

(b)

Fig. 10. (a) En face images selected per every eight frames from FD-DHOCI images of an $800 \mu \mathrm{m}$ diameter healthy rat tumor spheroid. (b) Pseudo-B-scans selected per every 11 frames from the data cube of the tumor shown in (a). 
with an $800 \mu \mathrm{m}$ diameter. Images are shown on a log reflectance scale. The tumor was attached to the bottom of a Petri dish with the light incident from the bottom of the petri dish. The bright specular reflection from the surface of the Petri dish was avoided by tilting it. The healthy tissue at the surface of the tumor presents a dim reflection, whereas the features deeper inside the tumor are brighter, especially at depths near frame 56. The stack of en face holographic images forms a data cube that can be visualized by the use of computed reconstruction. Figure 10(b) shows side views (pseudo-B-scans) of the tumor shown in Fig. 10(a). Cross sections in the $y-z$ plane were selected per every 11 frames from the data cube. The shape of the tumor spheroid is circular. Frame 98 is the approximate midsection. Figure 11 shows the volumetric visualization of the tumor spheroid from a cut-away volume to enhance the visibility of a 3D structure.

Figure 12(a) shows a collection of 400 pseudo-Ascans (reflectivity versus depth along selected curves) that were selected in the center area of $100 \mu \mathrm{m}$ $\times 100 \mu \mathrm{m}$ from the holographic en face images of a $1.4 \mathrm{~mm}$ diameter rat tumor. These data demonstrate that a penetration depth of $\sim 1.4 \mathrm{~mm}$ for the rat tumor can be achieved in FD-DHOCI. The graph shows that reflectivity increases to approximately a $200 \mu \mathrm{m}$ depth and then decreases exponentially. This reflectivity change with depth is related to the necrosis, which has higher reflectivity than the outer healthy shell (100-200 $\mu \mathrm{m}$ thick). Figure 12(b) shows the averages of 400 pseudo-A-scans that were selected in the center $100 \mu \mathrm{m} \times 100 \mu \mathrm{m}$ area from holographic en face images for tumors with 700, 850, 1100, and $1400 \mu \mathrm{m}$ diameters. The dashed curve in Fig. 12(b) is the average of the pseudo-A-scans in Fig. 12(a). The characteristic in the change of reflectivity with depth can be understood through the average of the pseudo-A-scans. In the upper area of the healthy shell $(\sim 200 \mu \mathrm{m})$, all tumors show a similar trend with an increase in reflectivity. The trend in the decrease of reflectivity is similar in the lower area of healthy shell for 700, 850, and $1100 \mu \mathrm{m}$ diameter tumors, while this trend is different for the $1400 \mu \mathrm{m}$ diameter tumor. This is related to the size of the necrotic area and to multiple scattering.

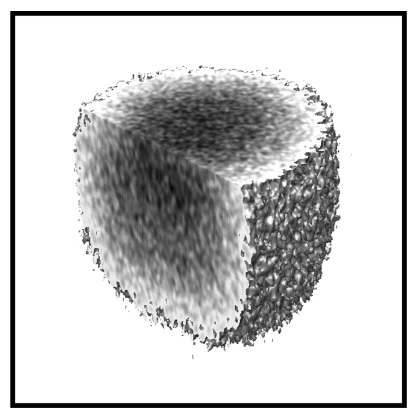

Fig. 11. Volumetric visualization, which is generated from the volumetric data cube of the tumor spheroid in Fig. 10.

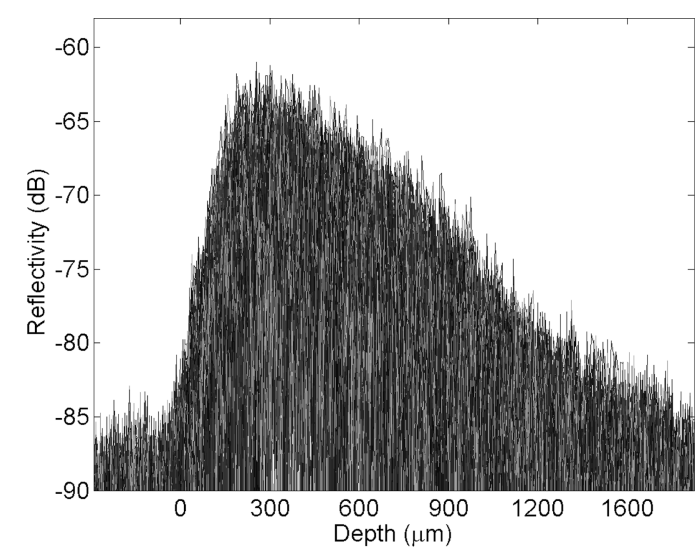

(a)

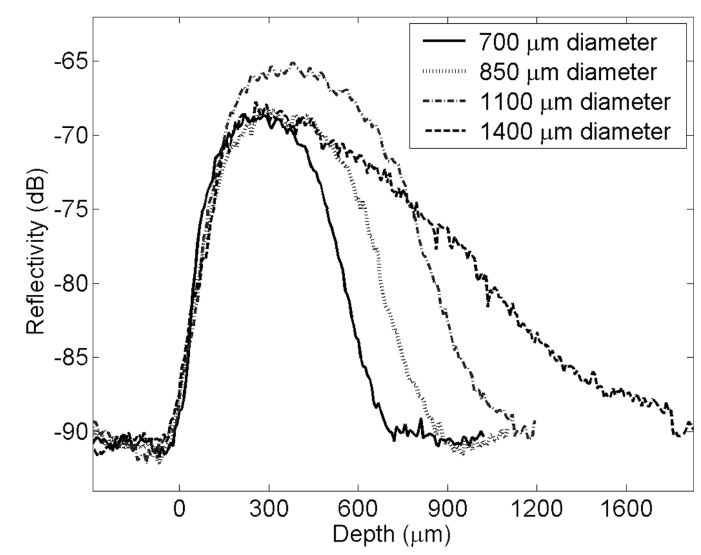

(b)

Fig. 12. (a) Collection of 400 pseudo-A-scans selected from en face holographic images of a $1.4 \mathrm{~mm}$ diameter rat tumor spheroid. (b) Averages of 400 pseudo-A-scans selected from holographic images of tumors with four different diameters. Dashed curve in (b) is the average of the pseudo A-scans in (a).

To demonstrate the general utility of FD-DHOCI, we used it to interrogate a whole mouse eye. The sample was prepared in vitro by detaching mouse eyes from the mouse postmortem, as shown in Fig. 13(a). The typical diameter of mouse eyes is $\sim 3 \mathrm{~mm}$. After capturing digital holograms with a depth step of $10 \mu \mathrm{m}$, digital holograms were Fourier-transformed and zero-order-subtracted. The medial section of the anterior segment of the mouse eye from a volumetric data cube is shown in Fig. 13(b). The section shows parts of the cornea and the iris in the mouse eye. The anterior chamber cornea-iridial angle is well observed with an angle of $20^{\circ}$. This anterior chamber angle is an indicator of glaucoma but is difficult to measure with conventional techniques. The diameter of the illumination on the sample is $\sim 800 \mu \mathrm{m}$ in diameter in the current experimental setup, and hence, the whole area of the anterior segment could not be captured from one fly-through. To capture the entire anterior segment of the mouse eye, ten fly-throughs were performed, and a mosaic section from the ten fly-throughs is shown in Fig. 13(c). The cornea, the 


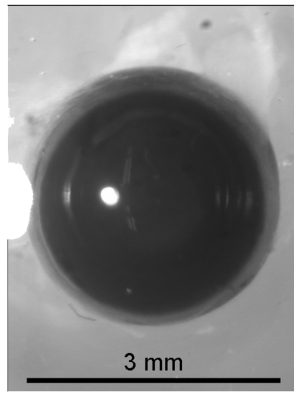

Sample

(a)

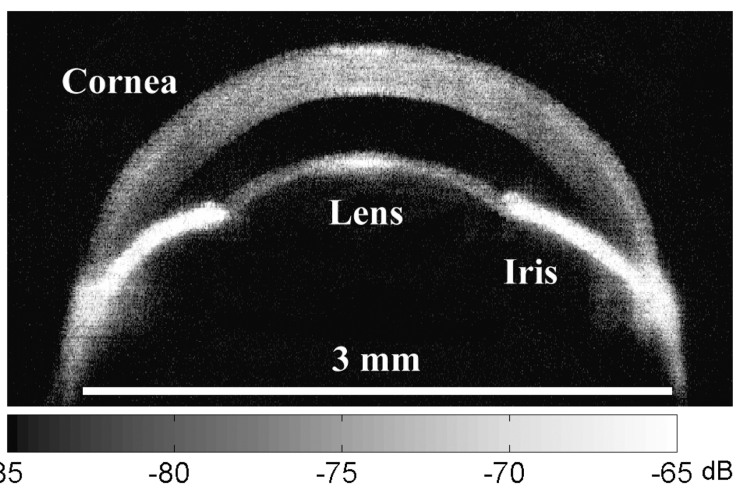

(c)

Fig. 13. (a) Sample of mouse eye in vitro (microscope image). (b) Section extracted from the FD-DHOCI volumetric data (from one fly-though) of a mouse eye, showing the cornea-iridial angle of $20^{\circ}$. (c) Mosaic section of the anterior segment extracted from ten flythroughs.

iris, and the lens in the mouse eye are well discernable in the section.

Imaging of retinal structure is clinically relevant for the diagnosis of a variety of diseases such as glaucoma, macular degeneration, and macular edema. We applied FD-DHOCI to image the mouse retina. The medial section image for a region of the mouse retina and sclera is shown in Fig. 14. The first refractive interface posterior to the lens represents the border between the lens and the vitreous humor. The second refractive interface shows the top of the ganglion cell layer. The structure of the optic disk is shown on the left, and the structure of retinal pigment epithelium, choroid, and sclera is at the bottom. It was difficult to observe retinal structure such as inner plexiform layer, inner nuclear layer, and outer nuclear layer in mouse retina in the current FDDHOCI system because of its limited resolution. However, the longitudinal resolution in our system can be improved by using a shorter-coherence length at the cost of lower sensitivity, and the transverse resolution can be improved by using a lens with higher NA and using a larger CCD chip at the cost of shorter depth of focus. We expect that retinal structure would be observed when the FD-DHOCI system with higher resolution is used.

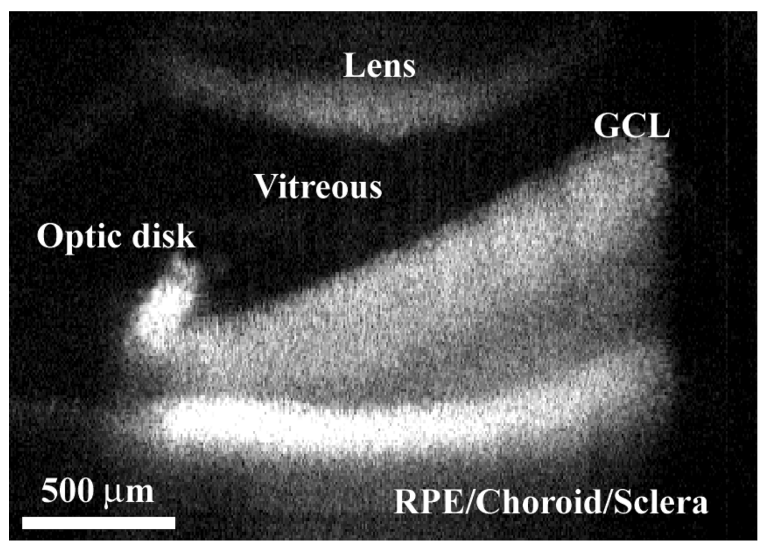

Log reflection

Fig. 14. Section of the retina of a mouse eye from FD-DHOCI volumetric data. GCL, ganglion cell layer; RPE, retinal pigment epithelium.

\section{Conclusion}

In conclusion, we have shown that Fourier-domain digital holographic optical coherence imaging (FDDHOCI) is capable of depth-resolved imaging inside tissue with a sensitivity of $86 \mathrm{~dB}$. The algorithm for numerical reconstruction is simply the fast Fourier transform of digital holograms, which is simple and permits fast reconstruction. We developed a zeroorder subtraction method by subtracting the average of the pixels spanning a single fringe spacing. A transverse resolution of $9 \mu \mathrm{m}$ and longitudinal resolution of $18 \mu \mathrm{m}$ were achieved in FD-DHOCI with an object intensity of $5 \mathrm{~mW} / \mathrm{mm}^{2}$ and an exposure time of $10 \mathrm{~ms}$. To the best of our knowledge, we present the first results of FD-DHOCI applied to rat tumor spheroids and mouse eyes. A penetration depth of $1.4 \mathrm{~mm}$ for a rat tumor spheroid was achieved. The images of the anterior segment and the retina in the mouse eye demonstrate potential utility for FD-DHOCI.

This work was supported by the National Science Foundation under grant BES-0401858.

\section{References}

1. K. A. Stetson, "Holographic fog penetration," J. Opt. Soc. Am. 57, 1060-1061 (1967).

2. K. G. Spears, J. Serafin, N. H. Abramson, X. Zhu, and H. Bjelkhagen, "Chronocoherent imaging for medicine," IEEE Trans. Biomed. Eng. 36, 1210-1214 (1989).

3. A. V. Mamaev, L. I. Ivleva, N. M. Polozkov, and V. V. Shkunov, "Photorefractive visualization through opaque scattering media," in Conference on Lasers and Electro-Optics, Vol. 11 of OSA Proceedings Series (Optical Society of America, 1993), pp. 632-634.

4. S. C. W. Hyde, N. P. Barry, R. Jones, J. C. Dainty, and P. M. W. French, "Sub-100 $\mu \mathrm{m}$ depth-resolved holographic imaging through scattering media in the near infrared," Opt. Lett. 20, 2330-2332 (1995).

5. S. C. W. Hyde, R. Jones, N. P. Barry, J. C. Dainty, P. M. W. French, K. M. Kwolek, D. D. Nolte, and M. R. Melloch, "Depthresolved holography through turbid media using photorefraction,” IEEE J. Sel. Top. Quant. Electron. 2, 965-975 (1996).

6. P. Yu, M. Mustata, L. Peng, J. J. Turek, M. R. Melloch, 
P. M. W. French, and D. D. Nolte, "Holographic optical coherence imaging of rat osteogenic sarcoma tumor spheroids," Appl. Opt. 43, 4862-4873 (2004).

7. K. Jeong, L. Peng, J. J. Turek, M. R. Melloch, and D. D. Nolte, "Fourier-domain holographic optical coherence imaging of tumor spheroids and mouse eye," Appl. Opt. 44, 1798-1805 (2005).

8. M. Tziraki, R. Jones, P. M. W. French, M. R. Melloch, and D. D. Nolte, "Photorefractive holography for imaging through turbid media using low coherence light," Appl. Phys. B 70, 151-154 (2000).

9. R. M. Brubaker, Q. N. Wang, D. D. Nolte, E. S. Harmon, and M. R. Melloch, "Steady-state four-wave mixing in photorefractive quantum wells with femtosecond pulses," J. Opt. Soc. Am. B 11, 1038-1044 (1994).

10. M. A. Kronrod, L. P. Yaroslavski, and N. S. Merzlykov, "Computer synthesis of transparency holograms," Sov. Phys.-Tech. Phys. 17, 329-332 (1972).

11. M. A. Kronrod, N. S. Merzlykov, and L. P. Yaroslavski, "Reconstruction of holograms with a computer," Sov. Phys.-Tech. Phys. 17, 333-334 (1972).

12. U. Schnars and W. P. O. Jüptner, "Direct recording of holograms by a CCD-target and numerical reconstruction," Appl. Opt. 33, 179-181 (1994).

13. U. Schnars and W. P. O Jüptner, "Direct recording and numerical reconstruction of holograms," Meas. Sci. Technol. 13, R85-R101 (2002).

14. I. Yamaguchi, J. Kato, S. Ohta, and J. Mizuno, "Image formation in phase-shifting digital holography and applications to microscopy," Appl. Opt. 40, 6177-6186 (2001).

15. E. Cuche, F. Bevilacqua, and C. Depeursinge, "Digital holography for quantitative phase-contrast imaging," Opt. Lett. 24, 291-293 (1999).

16. E. Cuche, P. Marquet, and C. Depeursinge, "Simultaneous amplitude-contrast and quantitative phase-contrast microscopy by numerical reconstruction of Fresnel off-axis holograms," Appl. Opt. 38, 6994-7001 (1999).

17. C. Wagner, W. Osten, and S. Seebacher, "Direct shape measurement by digital wavefront reconstruction and multiwavelength contouring," Opt. Eng. 39, 79-85 (2000).

18. B. Javidi and T. Nomura, "Securing information by use of digital holography," Opt. Lett. 25, 28-30 (2000).

19. F. Charrière, A. Marian, F. Montfort, J. Kuehn, T. Colomb, E. Cuche, P. Marquet, and C. Depeursinge, "Cell refractive index tomography by digital holographic microscopy," Opt. Lett. 31, 178-180 (2006).
20. G. Pedrini and S. Schedin, "Short coherence digital holography for 3D microscopy," Optik 112, 427-432 (2001).

21. L. Martínez-León, G. Pedrini, and W. Osten, "Applications of short-coherence digital holography in microscopy,” Appl. Opt. 44, 3977-3984 (2005).

22. C. Yuan, H. Zhai, X. Wang, and L. Wu, "Lensless digital holography with short-coherence light source for three-dimensional surface contouring of reflecting micro-object," Opt. Commun. 270, 176-179 (2007).

23. P. Massatsch, F. Charrière, E. Cuche, P. Marquet, and C. D. Depeursinge, "Time-domain optical coherence tomography with digital holographic microscopy," Appl. Opt. 44, 1806$1812(2005)$

24. L. Yu and M. K. Kim, "Variable tomographic scanning with wavelength scanning digital interference holography," Opt. Commun. 260, 462-468 (2006).

25. F. Montfort, T. Colomb, F. Charrière, J. Kühn, P. Marquet, E. Cuche, S. Herminjard, and C. Depeursinge, "Submicrometer optical tomography by multiple-wavelength digital holographic microscopy,” Appl. Opt. 45, 8209-8217 (2006).

26. K. Jeong, L. Peng, D. D. Nolte, and M. R. Melloch, "Fourierdomain holography in photorefractive quantum-well films," Appl. Opt. 43, 3802-3811 (2004).

27. K. Y. T. Seet, P. Blazkiewicz, P. Meredith, and A. V. Zvyagin, "Optical scatter imaging using digital Fourier microscopy," J. Phys. D: Appl. Phys. 38, 3590-3598 (2005).

28. P. Blazkiewicz, M. Gourlay, J. R. Tucker, A. D. Rakic, and A. V. Zvyagin, "Signal-to-noise ratio study of full-field Fourierdomain optical coherence tomography," Appl. Opt. 44, 77227729 (2005).

29. E. A. Swanson, D. Haung, M. R. Hee, and J. G. Fujimoto, "High-speed optical coherence domain reflectometry," Opt. Lett. 17, 151-153 (1992).

30. M. Born and E. Wolf, Principles of Optics, 6th ed. (Pergamon, 1980).

31. M. Tziraki, R. Jones, P. French, D. Nolte, and M. Melloch, "Short-coherence photorefractive holography in multiplequantum-well devices using light-emitting diodes," Appl. Phys. Lett. 75, 1363-1365 (1999).

32. L. A. Kunz-Schughart, M. Kreutz, and R. Knuechel, "Multicellular spheroids: a three-dimensional in vitro culture system to study tumour biology," Int. J. Exp. Pathol. 79, 1-23 (1998).

33. K. Groebe and W. Mueller-Klieser, "On the relation between size of necrosis and diameter of tumor spheroids," Int. J. Radiat. Oncol. Biol. Phys. 34, 395-401 (1996). 